\title{
Thyroid Gland Microfollicular Adenoma
}

National Cancer Institute

\section{Source}

National Cancer Institute. Thyroid Gland Microfollicular Adenoma. NCI Thesaurus. Code C4160.

A thyroid gland adenoma composed of microfollicular structures. 